\title{
Effects of modality preferences on learning and recall of bisensory stimuli*
}

\author{
GARY M. INGERSOLL \\ Indiana University, Bloomington, Indiana 47401 \\ and \\ FRANCIS J. DI VESTA \\ The Pennsylvania State University, University Park, Pennsylvania 16802
}

\begin{abstract}
The performance of visual attenders and aural attenders, as defined by performance on a bisensory digit-span task, was compared on three bisensory tasks. First, in replication of an earlier study, performance was compared on a bisensory missing-unit paradigm, in which $\mathrm{S}$ was required to respond with missing units from two independent sets of stimuli, each set being presented on a separate channel. The results corroborated those of an earlier study in which an interaction between modality preferences and presentation modality was found. Further, differential recall patterns were found for each of the groups. While such comparable results were not found on the other two tasks, differential performance on a bisensory paired-associate task and a bisensory connected discourse task were found. The results are interpreted in accord with Murdock's (1967) hypotheses concerning separate sensory storage mechanisms.
\end{abstract}

Studies of auditory and visual modality effects in short-term recall typically have been directed at comparing recall of material presented on one or the other of the two modes. Usually, an overall superiority of recall for auditory stimuli with accentuated recency effects is found in single modality presentation (e.g., Murdock, 1967; Murdock \& Walker, 1969). Similarly, overall superiority of recall for auditory stimuli has been found in studies of bisensory auditory-visual stimulation (Dornbush, 1968; Ingersoll \& Di Vesta, 1972). Murdock (1967; Murdock \& Walker, 1969) has also presented evidence in unisensory presentation to suggest that slightly greater primacy effects are usually found in recall of visual than in recall of auditory stimuli. The purpose of this study is to provide additional data on individual difference and modality effects in the bisensory situation that will be useful in testing models of human memory.

Far less research has been conducted using bisensory auditory-visual presentation than research comparing the efficacy of either modality singly. Mowbray (1952, 1953) presented two connected discourse passages simultaneously, one visually and one auditorily. Following the presentation, $\mathrm{S}$ was questioned to determine his level of comprehension of both passages. Mowbray concluded that the auditory presentation suffered more from simultaneous presentation than did the visual. Further, Mowbray argued that the easy passages suffered more during simultaneous presentation

*This study was partially supported by the Advanced Research Projects Agency (ARPA No. 1269), Office of Naval Research, Grant No. N00014-67-A-0385-006, to the second author. The authors are indebted to Harold Adams, Principal, Patrick Kelly, Guidance Counselor, and the staff and students of the Bald Eagle-Nittany Area High School, Mill Hall, Pennsylvania, for their help and cooperation during the conduct of this study. than did the difficult passages.

Using a bisensory auditory-visual analog to a dichotic listening task, Broadbent (1956; Broadbent \& Gregory, 1961) found that some Ss showed stable tendencies to recall the set presented to one sense prior to the set presented to the other sense. Similar results have been found by Dornbush (1968) and Senf (1969; Madsen, Rollins, \& Senf, 1970).

There has been a noticeable lack of research investigating possible interactive effects of specific individual differences on recall of auditory and visual stimuli, particularly under conditions of bisensory stimulation. In an earlier investigation, Ingersoll and Di Vesta (1972), defining aural and visual attending preference by recall patterns on a bisensory digit-span task, found evidence to indicate that recall as a function of presentation modalities interacted with modality preferences. Further, the interaction met the conditions of disordinality since not only were the recall patterns of aural and visual attenders in opposite directions, but each of those patterns was statistically significant (see, e.g., Bracht, 1970). That is, using a bisensory auditory-visual adaptation of the missing-units paradigm described by Yntema and Trask (1963), aural attenders recalled more auditory than visual missing units while visual attenders recalled more visual than auditory missing units. Furthermore, differences were also found in the overall patterns of recall for each group. Thus, not only did aural attenders recall more aural stimuli, but this recall was characterized by marked recency effects. Conversely, not only did visual attenders recall more visual stimuli, but their recall was marked by primacy effects.

At that time, Ingersoll and Di Vesta argued that the study should be replicated with a more heterogeneous population than college students and with additional 
measures introduced. The use of a more heterogeneous group would allow the incursion of a wide range of cognitive variables which might affect performance. Further, replicability would imply that the construct of modality preferences was distributed across a wider range of S populations. In part, then, the present study was conducted to test the stability of the results found therein. The principal consideration remained, however, to compare the effects of modality preferences on the recall of nonredundant materials presented simul taneously over auditory and visual modalities.

\section{METHOD}

The present investigation consisted of two parts. A measure of visual or aural preference was used to predict performance on a set of independent tasks. A more complete description of the materials and procedures can be found in Ingersoll and Di Vesta (1972).

Performance on a bisensory digit-span task of the type described by Broadbent (1956; Broadbent \& Gregory, 1961) was used to determine whether a $S$ was an aural attender or a visual attender. Two digit spans were presented simultaneously; one digit span was presented visually, while a second, different digit span was presented aurally. Modality preference was defined by $S$ 's performance during the recall portion of five criterion trials. The $S$ who tended to recall more visually presented stimuli than aurally presented stimuli on a series of criterion trials was classified as a visual attender. Conversely, the $S$ who tended to recall more aurally presented than visually presented stimuli was classified as an aural attender.

\section{Bisensory Missing-Unit Task}

A task was presented to $S$ in which a bisensory modification of the missing-units paradigm, described by Yntema and Trask (1963), was used. In the Yntema and Trask procedure, five words were first presented to $S$. Then four of the five words were repeated in random order. In the present investigation, two independent sets of five words were presented simultaneously, one set visually and one set auditorily. Therefore, S's task was to respond with two missing units, one from the set presented visually and one from the set presented auditorily. Ten trials, in which words were dropped equally from each of the five serial positions, were administered. Performance of visual attenders and aural attenders on the missing-units task as a function of auditory and visual presentation modalities was compared. The two groups, aural attenders and visual attenders, served as between-Ss dimensions. The design was a factorial design with 2 between- and 2 by 5 within-Ss dimensions, where responses to auditory and visual stimulation over each of the five serial positions served as the within-Ss dimensions.

The materials and procedure used in the earlier study were supplemented by two additional bisensory tasks. They were designed to measure the relationship between modality preference and performance on tasks requiring different levels of cognitive processes.

\section{Bisensory Paired-Associate Learning}

A modified study-recall paired-associate (PA) procedure was used. The procedure for this bisensory PA task differed from the traditional PA paradigm since word pairs were presented in a bisensory fashion, i.e., one word was presented on one modality and its associate was presented simultaneously on the other modality. Neither dimension was defined temporally or spatially as the stimulus or response, and no indications were given in the instructions concerning which item should be construed as the stimulus or response. The $S$ was instructed to recall as much of the complete list as possible, either as complete pairs or parts of pairs. The list of seven bisensory pairs was presented to all $S$ s for nine trials. The words were presented in a different random order on each trial.

The data for auditory and visual responses and the number of pairs recalled were analyzed separately. The inclusion of the latter measure in the former analysis would have produced significant results due to factors unrelated to the independent variable since the number of correct pairs must always be equal to or less than the fewest number of correct responses from either modality by itself. The first analysis was a repeated measures design with 2 between-Ss variables and 2 by 9 repeated measures. The second analysis had 2 between-Ss and 9 repeated measures.

\section{Bisensory Connected Discourse}

Paragraphs of approximately equal length were presented simultaneously over visual and auditory modalities. The visual paragraph was presented on a typewritten page, which $S$ was to read while listening to the simultaneous presentation of a different paragraph on a tape playback. The written paragraph was given to $S$ face down and was not turned over until the auditory presentation began. Further, in order to maintain equivalent presentation times, $S$ was instructed to stop reading and turn the page face down at the conclusion of the auditory paragraph. This procedure was repeated for two independent sets of paragraphs whose content was previously rated as familiar or unfamiliar by a comparable $S$ population. During recall, $S$ was instructed to write as many facts as he could from both passages. Order of recall and format of recall was not considered important in the scoring procedure.

Procedurally, this task differed in one important respect from all the others. The paragraphs used in the visual presentation were shown in their entirety to $S$. Whereas the other tasks maintained successive visual presentation, this task did not. Modality preference served as the between-Ss dimension, while the two levels of familiarity of the content of the passages over the two modalities served as within-Ss dimensions.

\section{Materials}

\section{Bisensory Paired-Associate Learning}

High-frequency monosyllabic four-letter nouns that had high imagery and concreteness ratings were selected from the Paivio norms (Paivio. Yuille, \& Madigan, 1968). Words were assigned randomly to seven pairs.

\section{Connected Discourse}

Two paragraphs each were adapted from introductory college texts in art history and biology. The same author was used within a topic in order to maintain some stability of writing style of paragraphs across modalities. Word and noun counts were subjected to nonparametric analyses to determine whether the passages seemed unduly different from each other. None of these statistics indicated significant differences $(p>.20)$.

\section{Apparatus}

Visual stimuli were single items presented simultaneously by slides, and auditory stimuli were presented by tape recorder, as they were in Ingersoll and Di Vesta (1972). The connected discourse passages were presented either in oral fashion via a tape recorder or visually as a typed passage, using Letter Gothic print on an $8 \times 11$ sheet of white paper.

\section{Subjects}

Students from the 11 th and 12th grades at the Bald Eagle-Nittany High School, Mill Hall, Pennsylvania, served as Ss 
in this investigation. In all, $201 \mathrm{Ss}$ were selected randomly from all juniors and seniors at that school. One female $S$ was eliminated from the analysis for failure to follow directions. Due to the failure of some Ss to return for the second portion of the study, despite written requests by $E$, complete data were not available on 711 th grade and 712 th grade $S$ s.

\section{Procedures}

Upon arrival at the laboratory, Ss were seated, in groups no larger than four, behind a large table, in front of which was a translucent screen. On the screen was projected a 3 by 3 array of dots, which served as a focus pattern. After Ss were seated. they were instructed about the general purpose of the study and instructed that there were actually two parts to the experiment and that it would be necessary for them to return in 1 week to participate in the second part. The Ss were then given instructions for the modality preference task (cf. Ingersoll \& Di Vesta, 1972).

Following the modality preference test, one of the three experimental tasks was presented. The order of presentation was such that each of the three bisensory tasks described earlier was presented on the first day an equal number of times. Thus, unlike the earlier study, the missing-units task did not necessarily follow immediately the modality preference task.

\section{RESULTS}

It was hypothesized that the aural and visual modality preferences, as defined by the magnitude and direction of the differences between the number of auditory and visual items recalled on the test task, would interact with bisensory stimulation to affect the recall of visual and auditory stimuli. It was also hypothesized that individuals who were characterized by one or the other modality preference would accentuate differences in those recall patterns typically associated with auditory and visual recall: thus, as in the Ingersoll and Di Vesta (1972) study, it was expected that greater primacy effects would be obtained in the recall of visual stimuli by visual attenders than by aural attenders, while greater recency effects would be obtained in the recall of auditory stimuli by aural attenders than by visual attenders. Similar predictions were offered for the additional tasks, i.e., modality preferences and modes of presentation should have different effects on recall. Mowbray $(1952,1953)$ found that with connected discourse, recall was greatest for the visually presented material. Thus, recall on that task was expected to be marked by visual superiority.

\section{Analysis of Variance for the Missing.Units Task}

The design used here implied a mixed analysis of variance. Aural and visual attenders served as the between-Ss groups, while recall of missing units from each of the five serial positions for each of the two modalities of presentation served as within-Ss dimensions.

No differences in overall performance were found as a function of either modality preferences or presentation modalities $(p>.05)$. However, as predicted, performance as a function of the interaction of modality preferences with presentation modalities was significant, $F(1,94)=4.45, p<.05$

A one-tailed comparison of mean correct responses for visual recall $(\overline{\mathrm{X}}=.23)$ to auditory recall $(\overline{\mathrm{X}}=.14)$ for visual attenders yiolded $\mathrm{t}=1.68, \mathrm{df}=94, \mathrm{p}<.05$. while a similar comparison of auditory recall $(\overline{\mathrm{X}}=.22)$ with visual recall $(\overline{\mathrm{X}}=.18)$ for aural attenders yielded $t=$ $1.21, \mathrm{df}=94, \mathrm{p}<.10$. Both comparisons indicate that the results are in the predicted directions, although statistically significant differences in recall were found only for visual attenders.

Recall, as a function of the serial position of the missing units, interacted with modality preferences, though the effect was not significant $(p<.10)$. However, the three-way interaction of Modality Preferences by Mode of Presentation by Serial Position was significant $(p<.05)$. An examination of mean auditory and visual responses for each group across serial positions reveals the patterns depicted in Fig. 1 (left and right). As can be seen in that figure, visual attenders not only showed overall superiority of recall for visual stimuli as compared to auditory stimuli, but the recall pattern was marked by augmented primacy effects, as is reflected by performance on the first two serial positions. Conversely, aural attenders not only demonstrated overall superiority of recall for auditory stimuli as compared to visual stimuli, but their recall pattern was marked by augmented recency effects, as reflected in performance on the last two serial positions. A one-tailed comparison of visual $(\overline{\mathrm{X}}=.32)$ and auditory $(\bar{X}=.11)$ performance of visual attenders on the first two serial positions yielded $t=2.17, \mathrm{df}=376$, $\mathrm{p}<.05),{ }^{1}$ favoring visual recall. On the other hand, a one-tailed comparison of auditory $(\overline{\mathrm{X}}=.30)$ and visual $(\overline{\mathrm{X}}=.18)$ performance of aural attenders on the last two trials yielded $\mathrm{t}=2.06, \mathrm{df}=376, \mathrm{p}<.05$, favoring auditory recall.

Analysis of recall as a function of the interaction of Presentation Modality by Serial Position yielded $F(4,376)=7.47, p<.01$. A one-tailed comparison of correct visual responses $(\overline{\mathrm{X}}=.22)$ to correct auditory responses $(\overline{\mathrm{X}}=.16)$ for the first two positions yielded $\mathrm{t}=$ 1.32. $\mathrm{df}=376, \mathrm{p}<.10$, indicating that visual recall is greatest during the primacy portion of the curve. Auditory recall, however, is greater on the next to last position but not the last position $(\mathrm{p}>.05)$. Nonetheless. the $t$ test of recall of auditory stimuli $(\overline{\mathrm{X}}=.26)$ compared with the recall of visual stimuli $(\overline{\mathrm{X}}=.18)$ on the last two serial positions yielded $t=1.70, d f=376$. $p<.05$.

\section{Analysis of Variance for the Bisensory Paired-Associate Task}

A repeated measures design was used in this analysis. Aural and visual attenders served as the between-Ss groups, and responses to auditory and visual stimuli 

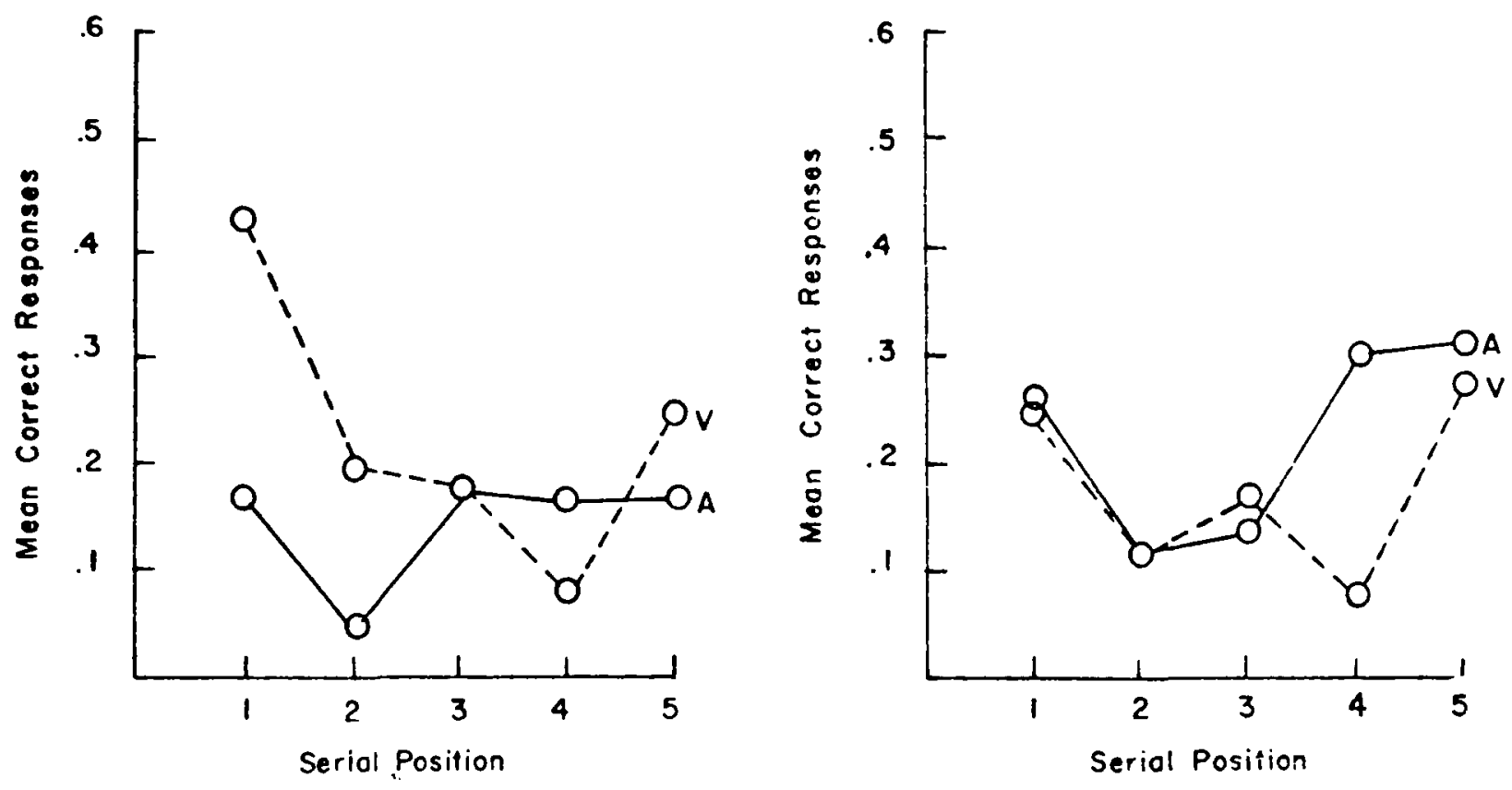

Fig. 1. (Left) Mean auditory (A) and visual (V) items correctly recalled by visual attenders. (Right) Mean auditory (A) and visual (V) items correctly recalled by aural attenders.

across nine trials served as the within-Ss dimension. The number of correct pairs across nine trials was analyzed separately. The inclusion of the latter measure in the first analysis would unduly inflate the results.

\section{Analysis of Auditory and Visual Responses}

No differences were found in performance as a function of modality preferences. However, recall of visual stimuli $(\overline{\mathrm{X}}=5.35)$ significantly $(\mathrm{p}<.01)$ exceeded the recall of auditory stimuli $(\overline{\mathrm{X}}=5.00)$. Exploratory comparisons of aural and visual recall, in blocks of three trials, were made via Scheffé's test for multiple comparisons. The comparison of visual to auditory recall for the first block of trials yielded $S^{2}=18.45, p<.05$. Similar comparisons for the second $\left(S^{2}=0.18\right)$ and third $\left(S^{2}=3.74\right)$ sets of trials failed to yield any differences in recall of visual and auditory stimuli. Thus, the greatest difference in recall of visual and auditory stimuli appears to have occurred in the first three trials. The differential responding across the three blocks of three trials offers an explanation of the interaction $(p<.01)$ between presentation modalities and trials. No variable interacted with modality preferences.

\section{Analysis of Correct Pairs}

The results of the analysis of the number of correctly identified pairs as a function of modality preferences shows that aural attenders correctly identified more pairs $(\overline{\mathrm{X}}=3.73)$ than did visual attenders $(\overline{\mathrm{X}}=1.53)$. This superiority was maintained across all trials. In addition, there was an interaction $(p<.001)$ between modality preference and trials. Analysis of the performance of the two groups across three blocks of three yielded $S^{2}=11.49, p<.01$, for Trials $1-3, S^{2}=$ $26.62, p<.001$, for Trials $4-6$, and $S^{2}=38.38$, $\mathrm{p}<.001$, for Trials 7.9 , suggesting that the superiority of recall for the aural attenders increased over trials.

\section{Analysis of Variance for the Bisensory Connected-Discourse Task}

The dependent measure for this task was the number of correct facts recalled for each of the connected-discourse passages. Both modality of presentation and the familiarity of the content of the passages served as the within-S dimensions. The two extreme groups of visual and aural at tenders were the between-Ss dimension. The design was thus a mixed design with 2 between-S and 2 by 2 within-Ss dimensions.

No overall difference was found favoring either group. Neither were any differences found as a function of familiarity of the passages. However, recall of facts from the visual passage $(\overline{\mathrm{X}}=3.18$ ) was greater than recall from the auditory passages $(\overline{\mathrm{X}}=2.31)$. Further, the superiority is concentrated in the recall of the low-familiarity passages, as reflected in the significant $(p<.01)$ interaction between presentation modality and the familiarity of the passages. Using Scheffé's test, it was demonstrated that visual recall $(\bar{X}=3.57)$ exceeded auditory recall $(\overline{\mathrm{X}}=1.70)$ on the low-familiarity passages. This comparison yielded $S^{2}=61.99, p<.01$. Recall as a function of modality fails to differ in the more familiar passages. 
One would expect that if modality preferences were to have any effect on the recall of facts for the passages there would be a parallel interaction between modality preferences and familiarity of the passages, i.e., visual attenders should show greater recall of the facts on the low-familiarity passages. This is, in fact, what occurred. Recall of facts as a function of modality preferences interacted significantly $(p<.05)$ with the familiarity of the presented passages. Following the presentation of the low-familiarity passages, visual attenders $(\overline{\mathrm{X}}=3.06)$ recalled more facts than did aural attenders $(\overline{\mathrm{X}}=2.46)$. The comparisons yielded $\mathrm{S}^{2}=7.36, \mathrm{df}=3.89, \mathrm{p}<.10$. No differences in recall were found between the two groups following the more familiar passages.

\section{DISCUSSION}

The results of this experiment unequivocally support the authors' earlier study. In some respects, they are more definitive, since the role of visual modality preferences and auditory modality preferences on the processing and storage of information presented visually and aurally was more clearly in accord with the predictions derived from a separate sensory storage model. Much of the research under the rubric of Aptitude by Treatment interactions remains tenuous: thus, replicability of the earlier study was important in light of the essentially new definition of modality preferences as the difference in recall of auditory and visual stimuli which had been presented simultaneously.

The results of the missing-units tasks serves first to corroborate the results of the earlier Ingersoll and Di Vesta (1972) study and, second, to offer empirical support for a separate sensory storage model of memory (Murdock, 1967). Further, the interaction of modality preferences with presentation modality under bisensory stimulation suggests that previous assumptions of overall auditory superiority during short-term recall are incomplete.

Murdock (1967), using a serial-probe paradigm with unisensory presentation, has repeatedly found an auditory superiority during recall. He has also found differential recall favoring visual stimuli during the primacy portion of the serial curve. Murdock interprets these data as supportive of a separate sensory storage model. However, the use of the serial-probe paradigm will lead to a truncation of primacy effects, since no viable probe occurs for the first item. In contrast, the missing-units paradigm used in this study assesses recall on that position. In the present study, primacy effects for visual presentations and recency effects over auditory presentations were found paralleling the effects demonstrated in unisensory auditory and visual short-term memory experiments. If the first serial position were not included in the missing-units task, a phenomenon which can be shown merely by eliminating the first part of the two recall curves, similar attenuation would have been found. The strongest support for
Murdock's model, however, is found in the performance of the visual attenders when compared to that of the aural attenders. As was expected, aural attenders displayed very strong recency effects in their recall of auditory stimuli and no difference in recall of visual and auditory stimuli in the primacy portion of the curve. This result, by itself, would not have been exceptionally supportive of a separate sensory store model, since that is essentially what has been found in the past. When, however, the results of the visual attenders are considered. strong contrast is found. Not only did visual attenders display greater recall of visual stimuli than of auditory stimuli, but their pattern of recall displayed augmented primacy effects favoring visual recall. Thus, while visual attenders displayed strong primacy effects during the recall of visual stimuli, aural attenders showed enhanced recency effects in their recall of auditory stimuli.

The recall patterns of the visual attenders offer strong contrast to those that would be predicted by an auditory storage buffer model such as the one proposed by Atkinson and Shiffrin (1968) and Phillips, Shiffrin, and Atkinson (1967). The stability of the results from this and the previous Ingersoll and Di Vesta (1972) study would suggest that the conventional buffer models must be considered as incomplete in their present form. The Phillips et al model suggests a probability function for the expulsion and loss of an item in the buffer on the presentation of new information. The greatest probability of expulsion is associated with the oldest item in buffer. Thus, the recall function for items from the buffer predicts that greatest recall is associated with the newest items in the buffer. While the model would appear valid across the entire population of Ss (especially for recall of auditory stimuli), the model is not adequate to predict the performance of the visual attenders.

The proposition might be introduced to suggest that visual attenders simply monitor information until the buffer is filled and then stop processing new information which would tend to induce an overflow within the buffer and require the expulsion of an existing item. If, however, this were the only factor affecting visual primacy, the Phillips et al model would predict that those items held in the buffer would still be liable to the same decay function. Thus, the serial position curve would show an increasing slope during the early trials. with a decrease in later trials. As can be seen in Fig. 1 (left), this is not the case. The slope of the initial points on the serial position continuum for visual recall was a negative function. Thus, it must be concluded that although the hypothesis of constricted input may partially account for the results, it is not a sufficient explanation. Conversely, aural attenders display recall patterns more in accord with predictions derived from the auditory buffer model. Concisely, the contrast supports a separate sensory storage model. It is evident that there are individuals who differentially prefer one 
or the other of the sensory stores and that these preferences act as monitoring filters during short-term recall.

The data from the bisensory paired-associate task revealed overall superiority of recall for visual stimuli over auditory stimuli, but show no differences between aural attenders and visual attenders on the recall of auditory and visual stimuli. However, large differences were found in the ability to associate the items as pairs. Further, that difference clearly favored the aural attenders. It is proposed that the nature of the task contributed in some way to the establishment of the visual items as the more salient. Thus, whereas no temporal or spatial component affected the definition of the nominal stimulus, the visual items probably acted as the functional stimuli. In terms of a two-stage model of paired-associate learning, the auditory stimulus would be processed as the response. Since aural attenders should have an advantage in storing the auditory stimuli, the problem of response integration would be reduced for the aural attenders, allowing them to concentrate on the establishment of the correct association. Thus, differences in performance in this type of paired-associate task were likely to appear in the associate stage of learning. Schulz (1969) has similarly concluded that differences due to modality of presentation are most likely to be found during the associative phase of learning.

The results of the bisensory connected discourse task present an enigma. While the data support the earlier research of Mowbray $(1952,1953)$, at least tentative evidence was found suggesting an interaction of modality preferences with presentation modalities. It is not clear, e.g., whether the salience of the visual stimulus was so strong that it, in effect, overshadowed the auditory or whether the results are contaminated by the availability of the visual stimulus for multiple readings. Clearly, more control is needed.

Finally, the conclusions of this and the previous Ingersoll and Di Vesta study offer interesting contrast to those of Laugherty and Fell (1969). While those authors found some evidence for $\mathrm{S}$ preferences for presentation modality, they were forced to conclude that short-term storage was primarily auditory. The discrepancy between the Laugherty and Fell conclusions and the conclusions of the present study may be due to the types of tasks used to assess differential performance as a function of modality preference, since the task demands were different in each.

\section{REFERENCES}

Atkinson, R. C., \& Shiffrin, R. M. Human memory: A proposed system and its control processes. In K. W. Spence and J. T.
Spence (Eds.), The psychology of learning and motivation: Advances in research and theory. Vol. 2. New York: Academic Press, 1968.

Bracht, G. Experimental factors related to aptitude-treatment interactions. Review of Educational Research, 1970, 40, 627-645.

Broadbent, D. E. Successive responses to simultaneous stimuli. Qiarterly Journal of Experimental Psychology, 1956, 8, 145-152.

Broadbent, D. E., \& Gregory, M. On the recall of stimuli presented alternately to the sense organs. Quarterly Journal of Experimental Psychology, 1961, 13, 103-109.

Dornbush, R. L. Input variables in bisensory memory. Perception \& Psychophysics, 1968, 4, 41-44.

Ingersoll, G. M., \& Di Vesta, F. J. Effects of modality preferences on performance on a bisensory missing-units task. Journal of Experimental Psychology, 1972, 93, 386-391.

Laughery, K. R., \& Fell, J. C. Subject preferences and the nature of information stored in short-term memory. Journal of Experimental Psychology, 1969, 82, 193-197.

Madsen, M. C., Rollins, H. A., \& Senf, G. M. Variables affecting immediate memory for bisensory stimuli: Eye-ear analogue studies of dichotic listening. Journal of Experimental Psychology, 1970, 83 (No. 3, Pt. 2).

Mowbray, G. H. Simultaneous vision and audition: The detection of elements running from overlearned sequences. Journal of Experimental Psychology, 1952, 44, 292-300.

Mowbray, G. H. Simultaneous vision and audition: The comprehension of prose passages with varying levels of difficulty. Journal of Experimental Psychology, 1953, 46, 365-372.

Murdock, B. B., Jr. Auditory and visual stores in short-term memory. Acta Psychologica, 1967, 27, 316-324

Murdock, B. B., Jr., \& Walker, K. D. Modality effects in free recall. Journal of Verbal Learning \& Verbal Behavior, 1969, 8, 665-676.

Paivio, A., Yuille, J. C., \& Madigan, S. A. Concreteness, imagery, and meaningfulness values for 925 nouns. Journal of Experimental Psychology, 1968, 76 (No. 1, Pt. 2).

Phillips, J. L., Shiffrin, R. M., \& Atkinson, R. C. Effects of list length on short-term memory. Journal of Verbal Learning \& Verbal Behavior, 1967, 6, 303-311.

Schulz, R. W. Learning of aurally received verbal material: Final report. Contract No. OE 5-10-018, University of Iowa, March 1969.

Senf, G. M. Development of immediate memory for bisensory stimuli in normal children and children with learning disorders. Developmental Psychology, 1969, 1, (No. 6, Pt. 2).

Yntema, D. B., \& Trask, F. P. Recall as a search process. Journal of Verbal Learning \& Verbal Behavior, 1963, 2, 65-74.

\section{NOTE}

1. The large degrees of freedom $(\mathrm{df}=376)$ associated with the $t$ tests presented here is the df for the error term on which the sampling fluctuation is based. The mean squared error is a pooled estimate of error across cells, and thus the df may be considerably greater than the $\mathrm{n}$ associated with any given cell.

(Received for publication February 12, 1973; revision accepted July 16,1973 .) 